\title{
Multiple seborrheic keratosis in a patient with familial benign pemphigus
}

\author{
Vera A. Smolyannikova, Alexandra K. Alexandrova
}

Department of Pathological Anatomy, Russian Ministry of Health I.M. Sechenov First Moscow State Medical University, Moscow, Russia

Corresponding author: Dr. Alexandra K. Alexandrova, E-mail: arfa27.80@mail.ru

\begin{abstract}
Familial benign pemphigus, also known as Hailey -Hailey disease (HHD), is a rare disease characterized by crusted erosions with marked predilection for intertriginous areas, especially the axillae, submammary and groin regions. Diagnosis and treatment are difficult. The article discusses the clinical features HHD in combination with multiple seborrheic keratosis (SK) on the background of insulin resistance of a 79 years old patient. Possible causes of the development of the SK are beeing discussed. The efficacy of topical and systemic antibiotic therapy was shown.
\end{abstract}

Key words: Familial benign pemphigus; Seborrheic keratoses; Insulin resistance; Therapy

\section{INTRODUCTION}

Hailey-Hailey disease (HHD) or familial benign chronic pemphigus is a dermatosis with an autosomal dominant inheritance characterized by full penetrance and variable expressivity: from individual resembling eczema lesions to extensive lesions of the skin. HHD causes mutations in the gene ATP2Cl, which encodes the ATPase calcium-magnesium secretory mechanism of a human $[1,2]$. The mechanism by which mutated ATP2Cl causes acantholysis, still not installed. Clinically, the disease manifests often at the age of 30-40 years in the form of painful erosions and scaly erythematous plaques in the intertriginous areas. Subjectively severe itching concerns, resulting in scratching and painful cracking. Conjunctiva, mucosa, and vulva involvement is rarely seen. Defeat folds accompanied by an unpleasant odor. After healing it is characterized by hyperpigmentation. The acute illness usually occurs under the influence of increased sun exposure, sweating, friction, irritating therapy. Remission can be from several months to several years. Histologically, HHD is similar to Darier disease: find suprabasal separation of the epidermis caused by acantholysis and resulting in lacunae or bullae, and upward proliferation of papillae as so-called villi into the lacunae.

Treatment HHD is an extremely complex task. Topical therapy using corticosteroid ointments in combination with systemic antibiotics gives temporary relief. Some reports have shown some benefit with retinoids, dapsone, cyclosporine, topical vitamin D analogs, and topical immunomodulators in recalcitrant cases. Their effectiveness, however, individual. Medical therapies generally do not induce prolonged remissions $[3,4]$.

The occurrence of multiple SK most authors consider as a sign of Leser - Trelat, is pretty rare dermatosis, signifying the presence or development of malignant tumors of internal organs. Despite the fact that large epidemiological studies have not reliably be attributed to a true paraneoplastic dermatoses, there were described many cases of its occurrence together with acanthosis nigricans, young adults, with internal malignant diseases $[3,5]$. In the literature we have not found cases of combining multiple SK and HHD, the following observation is presented.

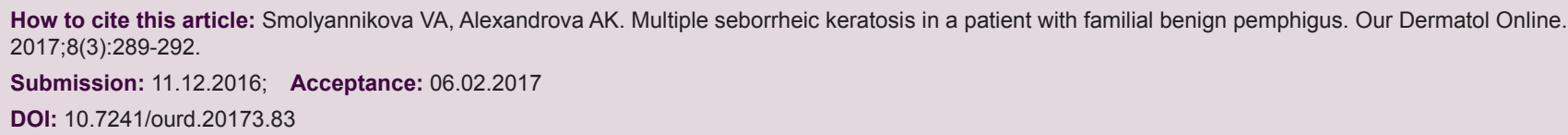




\section{CASE REPORT}

A 79 years- old woman presented to our dermatology clinic with a 8-year history of itching and pain at the eruption in the groin area, on the skin of the thighs, in the crotch. The first time skin rash groin area appeared in 2007, in the summer, a few weeks after surgery. Her medical history was characterized by hysterectomy for leiomyoma. The patient was recommended observation by the oncologist. Skin eruption smeared methyluracyl ointment, vaseline, occasionally she was troubled by itching. When she saw a dermatologist, it was diagnosed with a dermatophytic infection, however topical antifungal therapy has aggravated the skin and increased itching. Subsequent calls with diagnoses: contact dermatitis, eczema, also it was performed detoxication and desensitizing therapy: calcium gluconate $10 \%$, Na thiosulfate $30 \%$, antihistamines. Topical corticosteroid were administrated. Short-term effect of therapy followed by relapse. The therapy with oral prednisolone at a dose of $30 \mathrm{mg}$ with gradual reduction of the dose for 1 month also did not produce significant clinical improvements, in addition, a few weeks after the end of treatment, the eruption has spread to the inguinal - femoral folds on the inner thighs, the skin of the abdomen. Due to the lack of improvement the patient completely stopped treatment, she used to apply homeopathic ointment on her own on the rashes for several years. She was treated by fasting. Strong pain and itching in the lesions was noted in the summer. Also in the past 6-7 months it was noticed the first appearance single, and then multiple tumors on the skin of the abdomen, under the breasts, in the armpits, slow-growing and sometimes itchy.

From concomitant diseases: arterial hypertension of the 2nd degree, risk 3, impaired glucose tolerance (since 2015 year), obesity lst degree. In September 2015, conducted endoscopic polypectomy of the stomach.

She denies allergies to drugs, foods. A genetic predisposition has not been established due to the lack of reliable information about relatives.

Physical examination revealed the inflammatory process, with localized eruptions on the skin of the femoral - inguinal folds with the transition to the inner surface of the thighs, in the crease on the skin of the abdomen, in the perineum and around the anus (Fig. 1).

Presents erythematous patches with fine wet multiple erosions, covered with a grey, easily removable coating, with portions of vegetations on the internal skin surfaces of thighs, skin stagnant-red color, thinning, telangiectasia. Around the anus and in the perineum there are erythematous-squamous lesions covered with grayish-pink crusts, linear cracks, with an unpleasant odor. The nails are thinning and flake with distal edge. Hair is dull, grey. The presence of multiple SK different sizes draws attention from related skin pathology, with a tendency to merge, in the form of thin, as if glued to light-brown plaques. SK located in the folds under the breast, groin, in the large crease of the belly, the armpits, the individual elements are arranged on the skin side surfaces of the body (Fig. 2). There are also single fibroepithelial polyps on the skin of the chest and abdomen.

On the basis of anamnesis and the clinical picture was the preliminary diagnosis: Hailey-Hailey Disease (?). Multiple seborrheic keratoses (Leser - Trelat syndrome?).

To confirm the diagnosis with the informed consent of the patient there was conducted histological examination of skin biopsy material from the lesion in the groin area. Morphologic study revealed that on the background characteristic of seborrheic keratosis suprabasal had multiple lacunae and vesicles with the presence in the lumen of acantholytic cells. In a

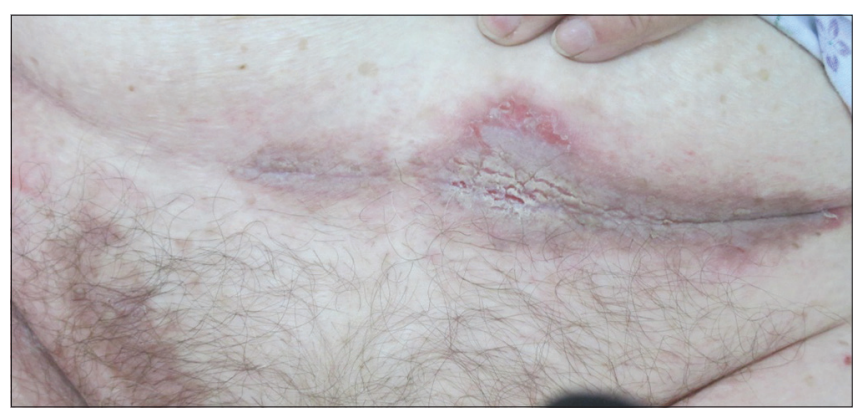

Figure 1: Clinical manifestations in patient with the HHD.

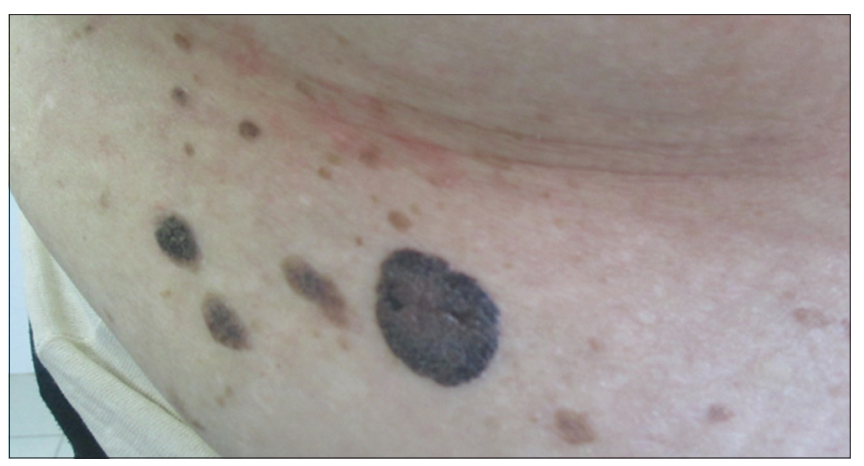

Figure 2: Multiple seborrheic keratosis in patient with the HHD. 
few areas there was registered villi, covered with the basal layer cells. Thus, histopathological examination confirmed the clinical diagnosis of HHD with central lesions seborrheic keratosis (Figs. 3 and 4).

Laboratory investigations showed elevated erythrocyte sedimentation rate $-35 \mathrm{~mm} / \mathrm{h}$; cholesterol $6.4 \mathrm{mmol} / \mathrm{l}$, capillary blood glucose $6.7 \mathrm{mmol} / \mathrm{l}$, glycated Hb-6,0.

A treatment with doxycycline 100mg 2 times daily, cetirizine dihydrochloride $10 \mathrm{mg} 1$ times daily for 10 days. Externally: metronidasole gel, gioxyson ointment, then clindamycin gel. Significant positive dynamics of the skin process was observed already at day 7 of therapy, seen after 21 days remained isolated erythematous-squamous lesions in the folds of the abdomen, the site of former lesions in the groin

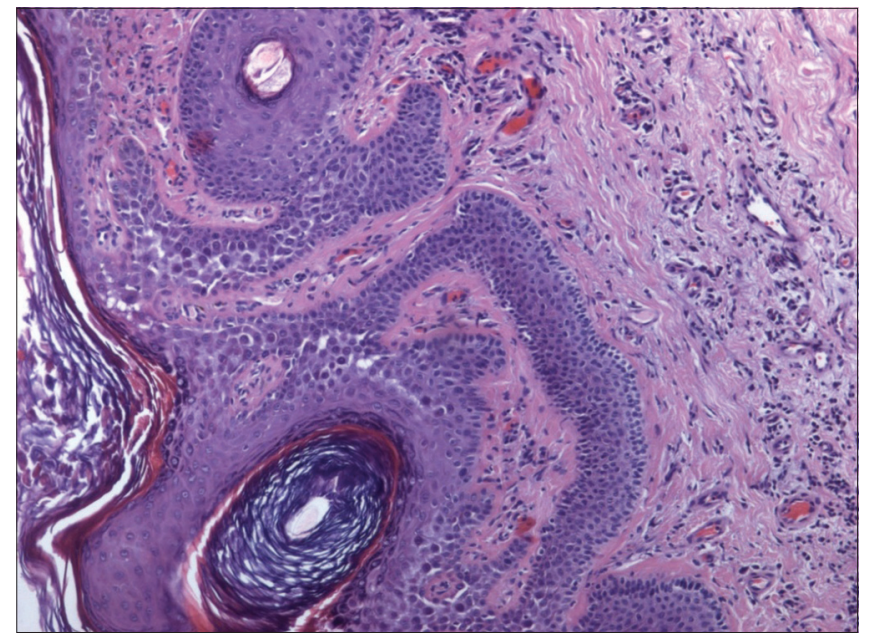

Figure 3: Histological picture of the patient with $\mathrm{HHD}$ and multiple SK (H\&E x100).

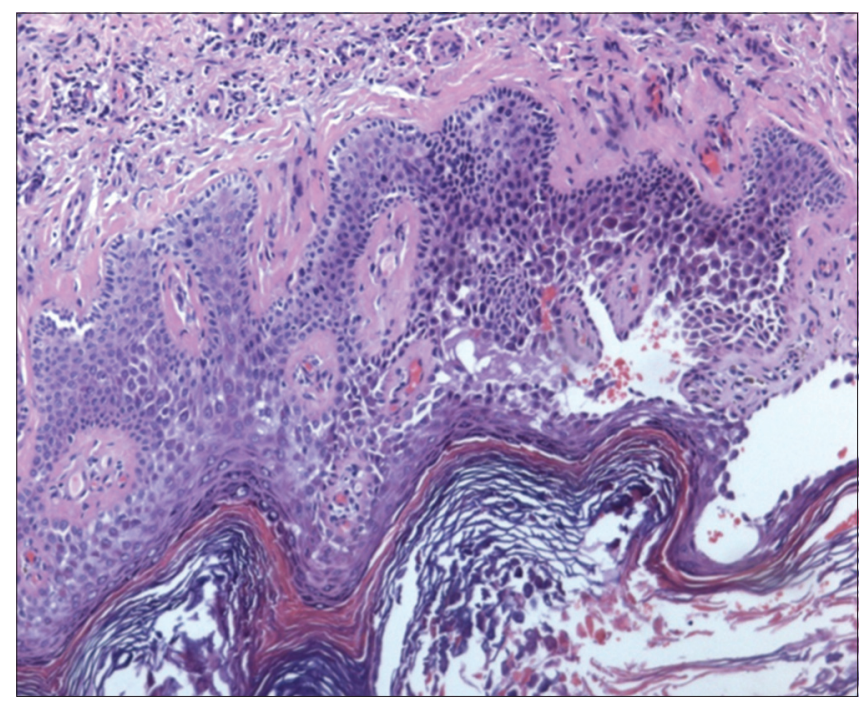

Figure 4: Histological picture of the patient with $\mathrm{HHD}$ and multiple SK (H\& E x100). area-areas of hyperpigmentation and multiple SK with inflammatory at the base.

\section{DISCUSSION}

On the basis of literature data the extreme variability of clinical manifestations BHH often leads to diagnostic errors, the disease is registered as eczema, bacterial or fungal infection $[3,6]$. We described clinical case which demonstrates once again the difficulty of diagnosis of chronic benign familial pemphigus Hailey-Hailey and the need of the confirmation morphologically. Pronounced positive effect of systemic and topical antibiotics and the ineffective of oral corticosteroid therapy is consistent with the data of other researchers about individual response to therapy of patients with PHH $[3,4]$.

Concomitant multiple SK arising in the patient mainly in the lesions (skin folds), can be explained by the development of impaired glucose tolerance. Thus, in the literature there is an observation of a patient with multiple SK on the background of diabetes mellitus type 2 . The author explains the development of multiple elements of SK for a short term effect of hyperinsulinaemia on the proliferative activity of keratinocytes [7]. In addition, earlier in our studies in 70 patients with multiple SK insulin resistance was detected in $85,7 \%$ cases [8].

However, given the presence in the patient of gastric polyps in the past year, the presence of an inflammatory response (increased erythrocyte sedimentation rate of $35 \mathrm{~mm} / \mathrm{h}$ ), we cannot exclude the presence of not identified malignant neoplasms of internal organs. It is known that the distribution of SK elements may contribute to the generation of malignant cells cytokines - endogenous mediators: transforming growth factor, epidermal growth factor and others, like insulin, affect the proliferation of keratinocytes and causes a rapid growth of SK lesions [9]. This hypothesis is confirmed by the study of Ellis DL. et al. In 1987 he identified the increase of the expression of epidermal growth factor receptors (EGF-R) in all epidermal layers, except the stratum corneum, in a patients with multiple SK, acanthosis nigricans and acrochordons [10].

Similar results were obtained by Ponti G et al. [5] in a patient with adenocarcinoma of the stomach and the syndrome of Leser-Trelat. Thus, the multiple eruptions of SK the patient in our clinical observation, can be considered in two ways: as a paraneoplastic process 
or as a consequence of insulin resistance and diabetes mellitus type 2 . The patient was prescribed supervision of a dermatologist, as well as consultation of the oncologist and endocrinologist.

\section{Consent}

The examination of the patient was conducted according to the Declaration of Helsinki principles. Written informed consent was obtained from the patient for publication of this article.

\section{REFERENCES}

1. Chao SC, Tsai YM, Yang MH. Mutation analysis of ATP2C1 gene in Taiwanese patients with HaileyHailey disease. Br J Dermatol. 2002;146:595.

2. Cialfi S, Oliviero C, Ceccarelli S, Marchese C, Barbieri L, Biolcati G, et al. Complex multipathways alterations and oxidative stress are associated with HaileyHailey disease. Br J Dermatol. 2010;162:518.

3. Wolff K, Goldsmith LA, Katz SI, et al. Fitzpatrick's Dermatology in General Medicine. McGraw-Hill Seventh edition; 2012. Chapter 49: 471-482
4. Hassan I, Keen A. Hailey Hailey disease: a case report. Our Dermatol Online. 2012;3:112-4.

5. Ponti G, Luppi G, Losi L, Giannetti A, Seidenari S. Leser-Trélat syndrome in patients affected by six multiple metachronous primitive cancers. J Hematology Oncol. 2010;3:2.

6. Zhang RZ, Zhu WY. Seborrheic keratoses in five elderly patient: an appearance of raindrops and streams. Indian J Dermatol. 2011;56:432-4.

7. Saraiya A, Al-Shoha A, Brodell RT. Hyperinsulinemia associated with acanthosis nigricans, finger pebbles, acrochordons, and the sign of Leser-Trélat. Endocr Pract. 2013;19:522-5.

8. Aleksandrova AK, Smolynnikova VA. Insulin resistance and seborrheic keratosis. J Dermatol Venereol. 2015: 5:25-8.

9. Heaphy MR Jr, Millns JL, Schroeter AL. The sign of Leser-Trelat in a case of adenocarcinoma of the lung. J Am Acad Dermatol. 2000;43:386-90.

10. Ellis DL, Kafka SP, Chow JC. Melanoma, growth factors, acanthosis nigricans, the sign of Leser-Trelat, and multiple acrochordons. A possible role for alpha-transforming growth factor in cutaneous paraneoplastic syndromes. N Engl J Med. 1987;317:1582-7.

Copyright by Vera A. Smolyannikova, et al. This is an open-access article distributed under the terms of the Creative Commons Attribution License, which permits unrestricted use, distribution, and reproduction in any medium, provided the original author and source are credited.

Source of Support: Nil, Conflict of Interest: None declared. 